\title{
Programa Mais Médicos: como avaliar o impacto de uma abordagem inovadora para superação de iniquidades em recursos humanos
}

\author{
Allan Claudius Queiroz Barbosa, ${ }^{1}$ Pedro Vasconcelos Amaral, ${ }^{2}$ Gabriel Vivas Francesconi, ${ }^{3}$ \\ Carlos Rosales, ${ }^{4}$ Elisandréa Sguario Kemper, ${ }^{3}$ Núbia Cristina da Silva, ${ }^{1}$ Juliana Goulart \\ Nascimento Soares, ${ }^{5}$ Joaquín Molina ${ }^{3}$ e Thiago Augusto Hernandes Rocha ${ }^{3}$
}

Como citar Barbosa ACQ, Amaral PV, Francesconi GV, Rosales C, Kemper ES, Silva NC, et al. Programa Mais Médicos: como avaliar o impacto de uma abordagem inovadora para superação de iniquidades em recursos humanos. Rev Panam Salud Publica. 2018;42:e185. https://doi.org/10.26633/RPSP.2018.185

RESUMO Apesar de decorridos 40 anos da divulgação dos princípios de Alma-Ata, ainda persistem desafios para a consolidação da atenção primária à saúde (APS) como eixo norteador dos sistemas de saúde ao redor do globo. Dentre os desafios ainda presentes, merecem destaque as questões associadas à iniquidade na distribuição de recursos humanos em saúde. A experiência do Programa Mais Médicos (PMM) no Brasil é um exemplo de proposta voltada para a abordagem dessa agenda inconclusa de Alma-Ata. Ao modificar aspectos centrais da formação, provimento e alocação de profissionais médicos, o PMM mostrou-se uma saída viável para minimizar os desafios de escassez de profissionais. As avaliações do PMM, embora incipientes, produziram evidências positivas quanto a ampliação do acesso e melhoria da qualidade da APS no Brasil, um país de médio desenvolvimento econômico. Apesar disso, é premente a geração de evidências mais sólidas a respeito do impacto do PMM sobre indicadores de desempenho da APS. O debate apresentado ao longo deste trabalho discute a necessidade de se viabilizar estudos quase-experimentais capazes de mensurar o impacto do PMM junto à saúde da população. O artigo propõe, então, um conjunto de diretrizes que pode se configurar como um modelo aplicável para abordar desafios associados à escassez de profissionais em países de médio e baixo desenvolvimento econômico.

Palavras-chave Recursos humanos; assistência à saúde; distribuição de médicos; alocação de recursos para a atenção à saúde.

Há 40 anos, a Declaração de Alma-Ata definiu as bases da atenção primária, elencando uma série de prioridades a serem abordadas para a superação dos desafios enfrentados pelos diferentes países no que tange às

Brasil. Correspondência: Thiago Augusto Hernandes Rocha, rochahernandes3@gmail.com

Universidade Federal de Minas Gerais, Departamento de Administração, Belo Horizonte (MG), Brasil.

2 Universidade Federal de Minas Gerais, Departamento de Economia, Belo Horizonte (MG), Brasil.

Organização Pan-Americana da Saúde/Organização Mundial da Saúde (OPAS/OMS), Brasília (DF),
Organización Panamericana de Salud/ Organización Mundial de Salud (OPS/OMS), San José, Costa Rica.

5 Universidade Federal de Juiz de Fora, Departamento de Administração, Juiz de Fora (MG), Brasil. questões relacionadas à saúde (1). Embora diversas nações tenham envidado esforços para operacionalizar os princípios da atenção primária à saúde (APS) defendidos em Alma-Ata, não foi possível, até o presente momento, superar a agenda definida em 1978 (2).

A despeito da impossibilidade de abordar, de modo satisfatório, os desafios que se faziam presentes em 1978, 
é importante reconhecer que o cenário atual difere daquele de Alma-Ata. Passados 40 anos, é possível observar inúmeras evidências sobre a efetividade e a eficiência da APS. Países com sistemas de APS fortes têm melhores indicadores de saúde a um menor custo (2). A diminuição de co-pagamentos, a ampliação do leque de serviços da APS, o alinhamento entre os serviços ofertados e as necessidades das populações estão associados a melhores desfechos de saúde (3).

Esses avanços foram expressivos, mas ainda há muito que fazer, especialmente no contexto dos países de baixo e médio desenvolvimento econômico (low and middle income countries, LMIC). Sem fortalecimento da APS, não é possível assegurar que os entraves atuais, relacionados a desempenho, baixa qualidade, falta de acesso, iniquidades, escassez de profissionais e desalinhamento entre o cuidado ofertado e as demandas da comunidade possam ser adequadamente abordados (4).

Para muitos LMIC, a temática da ampliação do acesso ainda é um ponto crítico. Esses países não conseguiram equacionar um suporte adequado para iniciativas de fortalecimento da APS (4). Dentre os fatores que contribuem para esse cenário estão a escassez de políticas públicas, baixa governança, crescimento populacional, sistemas de saúde inadequados, escassas avaliações de iniciativas da APS e ausência de políticas voltadas à gestão de recursos humanos em saúde (GRHS). A escassez de fundos de pesquisa contribui para agravar essa situação (4). Um trabalho de revisão apontou a falta de evidências rigorosas para apoiar a implantação de políticas de APS e o desenvolvimento de práticas de GRHS, especialmente em LMIC (5).

O presente trabalho pautou-se na agenda inconclusa de Alma-Ata e na premência de gerar evidências que abordem esses desafios. O trabalho enfoca uma iniciativa implementada com base na realidade brasileira, destinada à resolução de iniquidades na alocação e provimento de profissionais médicos: O Programa Mais Médicos (PMM). A estruturação do PMM tem-se mostrado uma alternativa viável à resolução de problemas que ainda se fazem presentes nas Américas e que remontam às discussões de Alma-Ata. Entretanto, a avaliação desse Programa tem-se mostrado um desafio no sentido da compreensão de quais indicadores efetivamente refletem o impacto do PMM na saúde da população: para um programa inovador é necessário identificar formas inovadoras de avaliação.

\section{INIQUIDADE NA DISTRIBUIÇÃO DE RECURSOS HUMANOS E ESTRATÉGIAS PARA SUPERAÇÃO - O PROGRAMA MAIS MÉDICOS}

A iniquidade na distribuição de profissionais médicos é um problema de escala global desde a década de 1960 (6). Além da má distribuição, há também o problema da escassez de médicos, especialmente em áreas mais vulneráveis e remotas (7). Essas questões têm afetado nações pobres e ricas e, até o presente momento, se configuram como um desafio não superado (8). A situação brasileira, até meados de 2013, não era diferente do panorama global.

Ao longo dos últimos anos, o Sistema Único de Saúde (SUS) brasileiro vem sendo reestruturado, com ações que priorizam a atenção básica (9). As iniciativas empreendidas visam a diminuir as vulnerabilidades a que certos grupos populacionais estão expostos (9). Apesar desses esforços, a questão do acesso ainda persiste como um problema crônico em determinadas localidades. Diante disso, o PMM foi desenhado como estratégia para repensar a lógica de formação e provimento de profissionais médicos, mediante uma parceria entre a Organização Pan-Americana da Saúde (OPAS), Ministério da Saúde do Brasil e governo de Cuba.

O PMM foi instituído em 2013 com o objetivo de aumentar a disponibilidade de profissionais médicos para o SUS, principalmente em áreas que apresentavam baixo número de médicos por habitante (6). Foram contemplados três eixos: mudança na matriz curricular dos cursos de medicina e ampliação de vagas; reaparelhamento das unidades básicas de saúde (UBS); e provimento emergencial de médicos através da contratação de médicos brasileiros, brasileiros formados no exterior e estrangeiros por um período de 3 anos (prorrogáveis por mais 3) para atuarem em localidades com escassez de profissionais (6). Até meados de 2015, havia 18240 profissionais atuando em $81 \%$ dos municípios brasileiros e a previsão de criação de 11,5 mil novas vagas de graduação em medicina (9).

Os três eixos que constituem o PMM, além de agregar medidas emergenciais de provisão de médicos para ampliar o acesso a serviços de saúde por parte de populações até então desassistidas, modificam, em médio e longo prazo, a estrutura de formação de médicos no Brasil (10). Ao reestruturar a forma como são alocados os profissionais e alterar a lógica de formação médica, o PMM coloca a provisão de médicos para a APS no centro da discussão política em saúde do país. Além disso, atribui à GRHS um papel norteador da política de APS brasileira.

Quase 5 anos após o início do PMM, surge agora a necessidade de se demonstrar a efetividade do programa quanto a questões como ampliação do acesso, melhoria da qualidade, mudança na lógica de formação de profissionais e impacto sobre indicadores de saúde populacional. O PMM é uma estratégia fundamental para a superação das iniquidades sociais e das desigualdades regionais no Brasil (11). Nesse sentido, a geração de evidências pode consolidar o PMM como uma via possível para abordar iniquidades com a potencialidade de aplicação em outras regiões das América.

\section{PREMÊNCIA NA GERAÇÃO DE EVIDÊNCIAS SOBRE OS RESULTADOS DO PMM}

A análise realizada, no presente artigo, quanto aos aspectos vinculados à GRHS no Brasil mostra uma tendência de mudança nos indicadores de recursos humanos em saúde. Por exemplo, para este trabalho, utilizou-se uma metodologia de clusters espaciais para analisar as taxas de rotatividade de profissionais médicos, a partir de dados do Cadastro Nacional de Estabelecimentos de saúde (CNES). Esse exercício evidenciou uma drástica redução na rotatividade médica em regiões que, antes da implantação do PMM, apresentavam valores elevados de rotatividade. Soma-se a isso a manutenção dos valores baixos de rotatividade para os anos seguintes (figura 1). Embora não tenham sido focados exclusivamente os municípios com PMM, a análise demonstra uma modificação no panorama do país após o início do programa.

Outro exemplo se debruçou sobre o tempo de permanência médio, em anos, dos profissionais médicos nas equipes de atenção básica. Nos anos que seguem a implantação do programa, houve uma 
FIGURA 1. Clusters espaciais de valores associados à rotatividade de profissionais médicos da atenção básica, Brasila
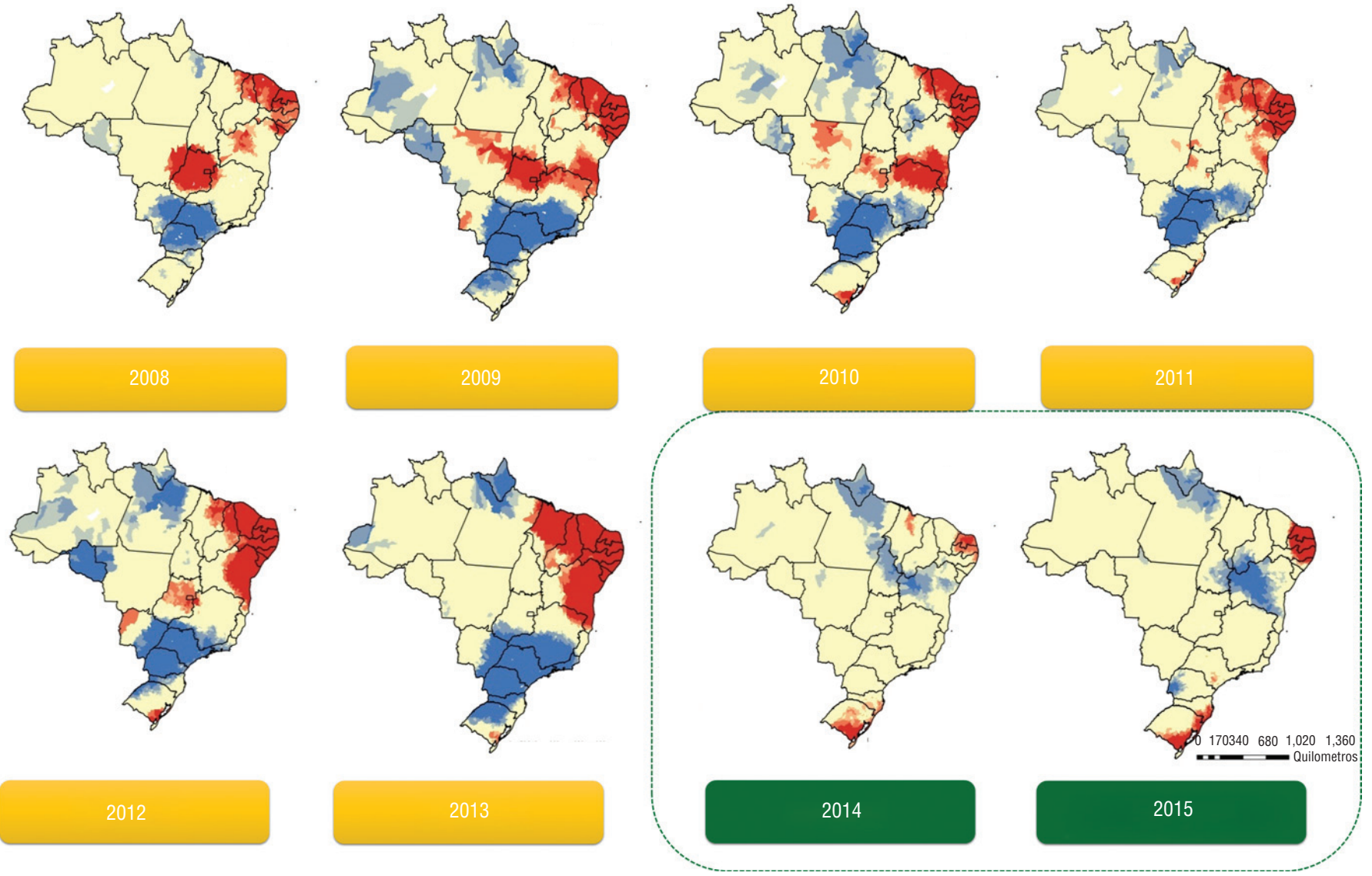

Fonte: elaborado pelos autores a partir de dados do Cadastro Nacional de Estabelecimentos de Saúde (CNES).

a Vermelho: clusters espaciais com alta rotatividade médica; azul: clusters com baixa rotatividade de médicos; amarelo: regiões que não foram categorizadas como pertencentes a clusters estatisticamente significativos.

inversão de tendência nos dados da série. Mesmo tomando as necessárias precauções para evitar uma associação espúria, é possível observar que o tempo de permanência médio dos médicos nas equipes da APS, que vinha em tendência de queda, a partir de 2014 se inverteu na maior parte das regiões brasileiras (figura 2).

A despeito dessas evidências mais gerais sobre o panorama de recursos humanos no Brasil, outros estudos se debruçaram sobre evidências associadas ao PMM. Tais trabalhos abordaram elementos associados a formação (12), satisfação do usuário (13), repercussão em relação ao volume de internações por condições sensíveis (ICSAP) (14), distribuição dos médicos (15), redução de iniquidades (6) e avaliações de desempenho utilizando abordagem quaseexperimental (16).

Para além dos resultados pontuais destacados, é preciso frisar a importância de trabalhos mais amplos de mapeamento de evidências. Um trabalho sobre panorama da produção científica associada ao PMM consolidou um levantamento sistematizado acerca dos resultados obtidos pelo PMM (17). Nessa publicação, foram identificados 82 trabalhos sobre o PMM. Desses, 54 trabalhos foram analisados quanto às categorias de análise de implantação, efetividade, mídia, limitações e críticas, formação e análise constitucional e jurídica (17). O panorama geral evidenciou que as avaliações do PMM são positivas, apontando resultados satisfatórios em relação a ampliação do acesso, equidade, satisfação dos usuários, humanização do cuidado, aumento de cobertura da APS, ampliação da rede de assistência e efeito sobre grupos específicos de ICSAP (17).

Entretanto, o PMM ainda não gerou um grande volume de artigos publicados, especialmente para a avaliação de resultados efetivos (17). Parte da carência de estudos dessa natureza pode ser explicada em função do pequeno lapso temporal entre a implantação do programa e o tempo necessário para que seja possível observar efeitos sobre indicadores de saúde populacional. Assim, é válida a hipótese de que muitas pesquisas estejam em desenvolvimento ou que tenham sido concluídas recentemente e seus resultados não tenham sido publicados.

A complexidade do PMM e suas múltiplas nuances e facetas acabam por se refletir no processo de geração de evidências de impacto. Tasca e Pêgo destacam que, para uma avaliação adequada de iniciativas complexas como o PMM, não basta medir indicadores tradicionais, como o número de médicos ou a rotatividade. É preciso identificar outros tipos de resultados e práticas inovadoras que permitam qualificar a APS, e que tenham sido viabilizadas pelo PMM (18). Apesar dos inúmeros avanços pontuais produzidos pelos estudos até aqui apresentados, 
FIGURA 2. Tempo médio de permanência, em anos, dos profissionais médicos junto às equipes de atenção primária, Brasil

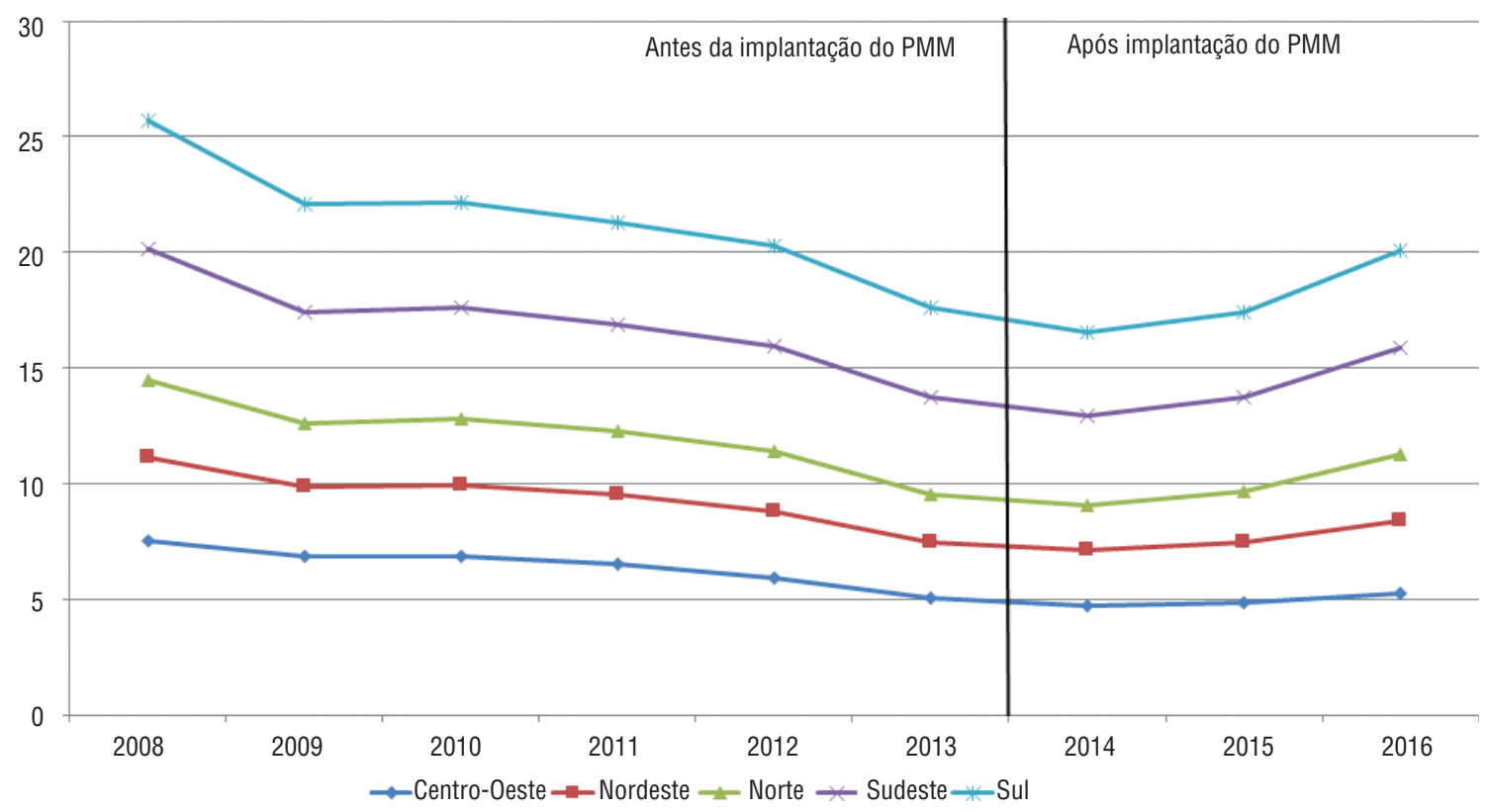

Fonte: elaborado pelos autores a partir de dados do Cadastro Nacional de Estabelecimentos de Saúde (CNES).

poucas foram as iniciativas com delineamento quase-experimental. Esse tipo de desenho metodológico permite avaliar qual parcela de um determinado resultado em saúde pode ser atribuída, exclusivamente, ao PMM.

\section{Uma proposta para avaliar o impacto do PMM e outras políticas públicas}

Os delineamentos quase-experimentais representam o melhor desenho metodológico disponível para avaliar políticas públicas, que raramente envolvem algum tipo de aleatorização no momento da implantação. Somente com esse tipo de abordagem é possível avaliar, satisfatoriamente, o impacto de políticas públicas - entendendo-se impacto como o efeito sobre uma variável de desfecho que pode ser atribuído a uma intervenção pregressa e cuja magnitude foi controlada pelo efeito de confundidores. A presente discussão oferece uma alternativa que permite abordar essa lacuna, apresentando uma abordagem sólida para a geração de evidências sobre o impacto de ações de recursos humanos em saúde.

Howard White (19) aborda os princípios básicos que permitem evidenciar o impacto de políticas públicas. Ele define os passos essenciais como: 1) mapear a cadeia causal; 2) compreender o contexto; 3) antecipar a heterogeneidade; 4) proceder uma avaliação rigorosa de impacto fazendo uso de contrafactuais; 5) realizar uma análise factual rigorosa; e 6) utilizar métodos e abordagens mistas. O desafio de avaliar políticas em saúde segundo esses princípios repousa na dificuldade de conseguir agregar evidências que permitam cumprir cada uma dessas etapas (20).

Considerando essa lacuna, pode-se procurar um parâmetro que permita aplicar o modelo de Howard White junto ao contexto do PMM. Mensurar o impacto de políticas implica comparar métricas de sujeitos que tenham sido expostos à intervenção com métricas de sujeitos que não tenham sido expostos à mesma intervenção. A complexidade do meio social atribui a essa tarefa nuances de inviabilidade. Assim sendo, é preciso mimetizar a situação, para ser possível identificar o impacto de políticas (20).

O mapeamento da cadeia causal de um programa (passo 1) pode ser feito pela análise detalhada de como a intervenção deve alcançar o impacto pretendido. No caso de políticas públicas como o PMM, isso pode ser feito através da análise de portarias, regulamentações e diretrizes gerais, que definem como seria esperado que a política funcionasse.
O passo 2 implica a compreensão de contexto de inserção da política. Nesse passo é preciso detalhar aspectos sociais, políticos e econômicos que possam modular os efeitos da política (20). No contexto específico do PMM, é preciso levar em conta aspectos sociodemográficos relacionados às regiões de inserção das equipes, perfil da população beneficiada e ajustar eventuais desfechos ao momento político de crise vivenciado pelo Brasil. Nesse ponto repousa um primeiro desafio operacional. As estatísticas vitais, os dados sociodemográficos e de nível de renda, por exemplo, somente são divulgados, anualmente, por município. Entretanto, em diferentes localidades de um mesmo município, por exemplo, Belo Horizonte, há bairros com índice de desenvolvimento humano (IDH) correspondentes à Suíça e à África do Sul, separados apenas por uma avenida (21). Assim, uma abordagem, para dar conta de contextualizar, adequadamente, os diferentes cenários necessários para permitir uma avaliação de impacto, precisa ser feita com a maior granularidade possível.

Outro desafio operacional está relacionado ao passo 3. A antecipação de heterogeneidade permite dimensionar variações no impacto de acordo com o desenho da intervenção, as características 
dos beneficiários ou o contexto socioeconômico e temporal (20). É preciso, então, desenvolver abordagens que deem conta de tratar com a maior granularidade possível as informações contextuais disponíveis para antecipar os efeitos de eventuais agentes confundidores que possam modular os resultados obtidos.

O segundo e terceiro passos são a base para definir o que está estipulado no passo 4 , ou seja, a avaliação satisfatória do impacto das políticas. A definição de contrafactuais é o que permite mimetizar a situação hipotética de comparação entre aqueles não expostos à intervenção frente àqueles expostos. Quanto melhor for a capacidade de definir contrafactuais rigorosos, melhores serão as chances de identificar diferenças pré e pós-exposição que sejam atribuíveis à política que se está avaliando (20). Para manejar os desafios entre os passos 2 e 4, foi desenvolvida uma metodologia mista para o estabelecimento de relações entre o PMM e desfechos em saúde, da forma mais granular possível para o contexto brasileiro.

Graças à disponibilidade de informações estruturadas, mesmo que com algumas fragilidades, em sistemas de informação em saúde, foi possível desenvolver uma abordagem inovadora para a definição de contrafactuais rigorosos para o PMM. A partir dos dados do Cadastro Nacional de Estabelecimentos de

FIGURA 3. Geolocalização das unidades básicas de saúde (UBS) que receberam profissionais do Programa Mais Médicos, Brasil, dezembro de 2015

Todas UBS com PMIM

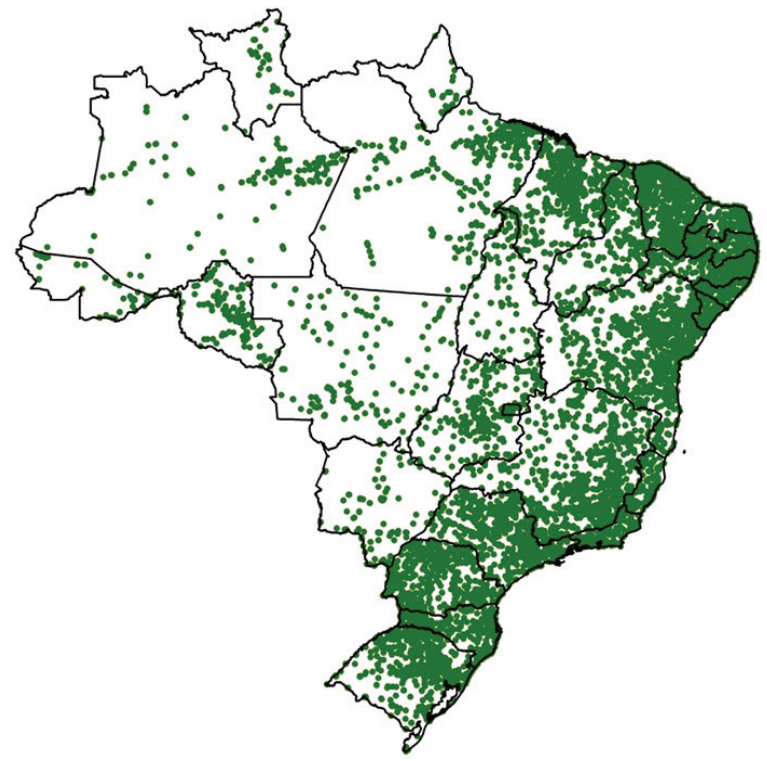

Fonte: Elaborado pelos autores a partir de dados do Cadastro Nacional de Estabelecimentos de Saúde (CNES).
Saúde (CNES), foi possível geolocalizar todas as equipes do país que receberam profissionais do PMM (figura 3).

A partir do mapeamento das unidades básicas de saúde (UBS), foram criadas áreas de abrangência potencial, refletindo uma porção de território de responsabilidade de cada UBS do país. Essa metodologia já foi utilizada no passado com relativo sucesso em um estudo destinado a avaliar a equidade no acesso a serviços de atenção básica (22). Uma vez definida essa área, é possível obter dados dos setores censitários incluídos em cada área potencial. A partir disso, é possível obter dados sobre saneamento básico, razão de sexos, nível de escolaridade, renda e composição familiar. Esse conjunto de dados é uma medida valiosa para cumprir o que está estipulado nos passos 2 e 3 do modelo de Howard White.

A medida de desfecho selecionada para se avaliar o impacto do PMM foram as ICSAP, uma vez que as mesmas se configuram como uma medida sintética e expressiva da qualidade dos serviços de atenção primária prestados à população (23). Apesar dessa vantagem, as ICSAP são divulgadas apenas de forma agregada pelo DATASUS, não permitindo, até recentemente, sua vinculação a equipes ou unidades de saúde. Para contornar essa dificuldade, Rocha et al. (24) desenvolveram uma abordagem de

geolocalização de ICSAP. A aplicação dessa abordagem conjuntamente com a definição de uma área de abrangência potencial das equipes torna possível atribuir a uma UBS uma carga de ICSAP. Um piloto dessa metodologia foi aplicado por Rocha et al. (24) junto às ICSAP processadas por hospitais brasileiros do estado de Goiás (figura 4).

O resultado da espacialização de ICSAP evidenciou diferentes padrões de agrupamento, o que destaca a importância da nova abordagem para atender os pressupostos estabelecidos por Howard White. Ao agregar informações dos setores censitários, das áreas de abrangência potencial, das equipes de atenção básica e das ICSAP geolocalizadas, torna-se possível separar equipes PMM das equipes não PMM para fins de comparação. Essa comparação pode ser feita de forma controlada pelos confundidores sociodemográficos, de renda e saneamento básico, de forma a mimetizar a situação de exposição ou não à intervenção. Como a série histórica das ICSAP cobre um período entre 2008 até 2018, é possível traçar uma linha de base pregressa ao PMM e acompanhar a sua evolução após a implantação do PMM.

Os passos 5 e 6 definidos pelo modelo de Howard White podem ser então implementados. Isso significaria uma sobreposição geográfica das informações sobre mapeamento das áreas de cobertura potenciais das UBS e geolocalização de ICSAPs. A análise factual rigorosa pode ser feita pela comparação de desfechos de equipes de atenção básica do PMM com equipes não PMM. O arcabouço de passos exemplificado à luz do PMM pode ser replicado para outras iniciativas voltadas para a atenção primária. A tradução do arcabouço aqui descrito para outros contextos nas Américas será balizada por especificidades locais, mas, uma vez respeitada a sequência de passos aqui detalhada, é possível delinear abordagens sólidas para a avaliação de impacto de políticas de GRHS.

\section{PERSPECTIVAS PARA AS AMÉRICAS}

O uso de metodologias quase-experimentais para a avaliação de políticas públicas é algo desejável, pois, em diversas circunstâncias, não é possível adotar delineamentos baseados em ensaios randomizados. Assim, as abordagens quase-experimentais se configuram como o melhor padrão possível para a avaliação 
FIGURA 4. Geolocalização de internações por condições sensíveis à atenção primária em hospitais de Goiás, Brasil, 2015

Doenças de imunização Gastroenterite infecciosa

Fonte: Elaborado pelos autores a partir de dados do Sistema de Informações Hospitalares (SIH).

de impacto de políticas públicas. Já existem recomendações específicas para a implantação de estudos avaliativos orientados segundo esses princípios (25). O uso de modelagens baseadas em ensaios com desenho stepped wedge tem ganhado popularidade graças à sua capacidade de abarcar as características das políticas públicas e, ainda assim, permitir delineamentos quase-experimentais.

Dal Poz et al. (26) conduziram um interessante estudo que avaliou os programas voltados para a GRHS nas Américas. Os autores destacam a fragmentação e a segmentação dos sistemas de saúde dos países latino-americanos e caribenhos. Tais características constituem uma barreira importante para ampliar a cobertura, alcançar cuidados de saúde primários integrados e reduzir a ineficiência e a descontinuidade dos cuidados (26). Destacaram ainda que os programas orientados para a GRHS, em 15 países das Américas,

1. Declaration of Alma-Ata. Lancet. 1979;1(8109):217-8.

2. Rawaf S, De Maeseneer J, Starfield B. From Alma-Ata to Almaty: a new start for primary health care. Lancet. 2008;372(9647):1365-7. enfrentam problemas comuns, como a falta de suporte político e financeiro (26). A despeito desses desafios, a saída apontada no estudo perpassa o intercâmbio de práticas, o planejamento prévio de ações, a avaliação de iniciativas e a disseminação de programas bem-sucedidos.

A abordagem destacada ao longo do presente trabalho aponta algumas medidas conceituais e de avaliação de impacto para o manejo dos desafios da GRHS. Destaca ainda a potencialidade do PMM em abordar desafios que persistiam há décadas. Sem prover a adequada distribuição de profissionais, com o perfil de formação correto e com a capacidade de ofertar cuidados de saúde onde os mesmos são necessários é impossível assegurar o pleno cumprimento dos parâmetros básicos definidos em Alma-Ata.

A abordagem adotada pelo PMM e o desenho avaliativo aqui ilustrado podem

\section{REFERÊNCIAS}

3. Starfield B, Shi L, Macinko J. Contribution of primary care to health systems and health. Milbank Q. 2005;83(3):457-502.

4. Walley J, Lawn JE, Tinker A, de Francisco A, Chopra M, Rudan I, et al. Primary ser adaptados para outras regiões das Américas que enfrentam iniquidade na distribuição de profissionais de saúde. $\mathrm{O}$ uso de metodologias mistas tem criado condições para superar os desafios vinculados ao delineamento de estudos quase-experimentais para avaliação de políticas. Os percalços superados pelo PMM podem servir de lição para o aprimoramento de práticas e contribuir para o arranjo mais eficaz de iniciativas públicas dedicadas a abordar a escassez de profissionais de saúde.

Conflitos de interesse. Nada declarado pelos autores.

Declaração. As opiniões expressas no manuscrito são de responsabilidade exclusiva dos autores e não refletem necessariamente a opinião ou política da RPSP/PAJPH ou da Organização PanAmericana da Saúde (OPAS). 
analysis of systematic reviews. Lancet. 2008;371(9613):668-74.

6. Carvalho VK da S, Marques CP, Silva EN da. A contribuição do Programa Mais Médicos: análise a partir das recomendações da OMS para provimento de médicos. Cienc Saude Colet. 2016;21(9):2773-84.

7. Dussault G, Franceschini MC. Not enough there, too many here: understanding geographical imbalances in the distribution of the health workforce. Hum Resour Health. 2006; $4: 12$

8. World Health Organization (WHO). Increasing access to health workers in remote and rural areas through improved retention: global policy recommendations. WHO; 2010. Disponível em: http://www. searo.who.int/nepal/mediacentre / 2010_increasing_access_to_health_workers_in_remote_and_rural_areas.pdf. Acessado em 24 de setembro de 2018.

9. Campos GW de S, Pereira Júnior N. A atenção primária e o programa mais médicos do Sistema Único de Saúde: conquistas e limites. Cienc Saude Colet. 2016;21(9):2655-63.

10. Minayo MC, Gualhano L. Pesquisas sobre o programa mais médicos: análises e perspectivas. Cienc Saude Colet. 2016; 21(9). Disponível em: http://www.scielo.br/scielo.php?pid=S1413-812320160 $11000001 \&$ script $=$ sci_arttext_pr\&tlng $=$ pt Acessado em 24 de setembro de 2018.

11. Travassos C, Oliveira EXG de, Viacava F. Desigualdades geográficas e sociais no acesso aos serviços de saúde no Brasil: 1998 e 2003. Cienc Saude Colet. 2006;11(4):975-86.

12. Thume E, Wachs LS, Soares MU, Cubas MR, Fassa MEG, Tomasi E, et al. Reflexões dos médicos sobre o processo pessoal de aprendizagem e os significados da especialização à distância em saúde da família. Cienc Saude Colet. 2016;21(9):2807-14.
13. Comes $Y$, Trindade J de S, Shimizu HE, Hamann EM, Bargioni F, Ramirez L, et al. Avaliação da satisfação dos usuários e da responsividade dos serviços em municípios inscritos no programa mais médicos. Cienc Saude Colet. 2016;21(9):2749-59.

14. Gonçalves RF, Sousa IMC de, Tanaka OY, Santos CR dos, Brito-Silva K, Santos LX, et al. Programa mais médicos no Nordeste: avaliação das internações por condições sensíveis à Atenção Primária à Saúde. Cienc Saude Colet. 2016;21(9):2815-24.

15. Nogueira PTA, Bezerra AFB, Leite AFB, Carvalho IM de S, Gonçalves RF, BritoSilva KS de. Características da distribuição de profissionais do Programa Mais Médicos nos estados do Nordeste, Brasil. Cienc Saude Colet. 2016;21(9):2889-98.

16. Santos LM, Oliveira A, Trindade JS, Barreto IC, Palmeira PA, Comes Y, et al. Implementation research: towards universal health coverage with more doctors in Brazil. Bull World Health Organ. 2017;95(2):103-12.

17. Kemper ES, Mendonça AVM, Sousa MF de. Programa mais médicos: panorama da produção científica. Cienc Saude Colet. 2016;21(9):2785-96.

18. Tasca R, Pêgo RA. Entrevista: avaliação de impactos do programa mais médicos: como medir os resultados? Cienc Saude Colet. 2016;21(9):2917-8.

19. White H. Theory-based impact evaluation: principles and practice. J Dev Effect. 2009;1(3):271-84.

20. Aquino R. Notas para discussão de uma proposta de avaliação de impacto do Projeto Mais Médicos para o Brasil PMMB. Salvador; 2017. Disponível em: https://simm.campusvirtualsp.org /sites/default/files/Notas sobre avaliaçao impacto PMM.pdf Acessado em 24 de setembro de 2018.
21. Fonseca B, Silva K. Atribuição de IDH aos bairros de Belo Horizonte. Transite. 23 de maio de 2017. Disponível em: http:/ / transite.fafich.ufmg.br/idh-bairros-de-belohorizonte/ Acessado em 5 de setembro de 2017.

22. Andrade MV, Noronha K, Barbosa AC, Rocha TA, Silva NC, Calazans JA, et al. A equidade na cobertura da Estratégia Saúde da Família em Minas Gerais, Brasil. Cad Saude Publica. 2015;31(6):1175-87.

23. Nedel FB, Facchini LA, Martín-Mateo M, Vieira LAS, Thumé E. Programa saúde da família e condições sensíveis à atenção primária, Bagé (RS). Rev Saude Publica. 2008;42(6):1041-52

24. Rocha TAH, Silva NC, Amaral PV, Vissoci JRN, Thomaz EBAF, Queiroz RCS, et al. Geolocalização de internações: uma solução baseada no programa estatístico $R$ para a implantação de possibilidades de análise baseadas no sistema de informação hospitalar. Belo Horizonte; setembro de 2017. Disponível em: http://www.cedeplar.ufmg.br/pesquisas/td/TD 567.pdf Acessado em 24 de setembro de 2018.

25. CIPHER Investigators. Supporting policy in health with research: an intervention trial (SPIRIT) - protocol for a stepped wedge trial. BMJ Open. 2014;4(7): e005293.

26. Dal Poz MR, Sepulveda HR, Costa Couto MH, Godue C, Padilla M, Cameron R, et al. Assessment of human resources for health programme implementation in 15 Latin American and Caribbean countries. Hum Resour Health. 2015;13:24.

Manuscrito recebido em 13 de janeiro de 2018. Aceito em versão revisada em 18 de setembro de 2018 .
ABSTRACT

More Doctors recruitment program: a new approach to overcome inequalities in human resources

Keywords
Despite the 40 years elapsed since the Alma-Ata principles were first launched, a series of challenges still persists for the consolidation of primary health care (PHC) as the backbone of health care systems around the world. Among these challenges, especially noteworthy are the issues associated with the inequality in the allocation of human resources. The experience of the More Doctors Program (Programa Mais Médicos, PMM) in Brazil is an example of initiatives that tackle this inconclusive AlmaAta agenda. By changing key aspects of physician training, provision, and allocation, PMM was shown to be a feasible alternative to minimize the challenge of physician shortage. Assessments of PMM, even though preliminary, have produced positive evidence showing increase in access and improvement of PHC quality in Brazil, a middle income country. Nevertheless, the generation of more robust evidence regarding the impact of PMM on PHC performance indicators is urgent. The discussion proposed in the present article emphasizes the need to prioritize quasi-experimental studies to measure the impact of PMM on population health. The article thus introduces a set of guidelines that may become a useful model to approach challenges associated with the shortage of health care professionals in low and middle income countries.

Human Resources; delivery of health care; physicians distribution; health care rationing. 
RESUMEN

Programa Más Médicos: cómo evaluar el impacto de un enfoque innovador para superar las inequidades en recursos humanos

Palabras clave
A pesar de que han transcurrido 40 años desde la proclamación de los principios de Alma-Ata, aún persisten desafíos para la consolidación de la atención primaria de salud (APS) como columna vertebral de los sistemas de atención de salud en todo el mundo. Entre estos desafíos, se destacan los problemas asociados con la desigualdad en la distribución de recursos humanos. La experiencia del Programa Más Médicos (PMM) en Brasil es un ejemplo de las iniciativas que abordan esta agenda inconclusa de Alma-Ata. Al cambiar aspectos clave de la capacitación, la provisión y la asignación de médicos, el PMM demostró ser una alternativa viable para minimizar el desafío de la escasez de profesionales. Las evaluaciones del PMM, aunque preliminares, han producido evidencias positivas que muestran un aumento en el acceso y mejora de la calidad de la APS en Brasil, un país de ingresos medios. Sin embargo, urge generar evidencia más sólida sobre el impacto del PMM en los indicadores de desempeño de la APS. La discusión propuesta en este trabajo enfatiza la necesidad de priorizar estudios cuasiexperimentales para medir el impacto del PMM en la salud de la población. El artículo propone un conjunto de directrices que pueden convertirse en un modelo útil para abordar los desafíos asociados con la escasez de profesionales de la salud en países de ingresos bajos y medios.

Recursos humanos; prestación de atención de salud; distribución de médicos; asignación de recursos para la atención de salud. 